\title{
Effects of density and anatomical feature on mechanical properties of various wood species in lateral tension
}

\author{
Yuka Miyoshi $^{1}\left[\right.$ Keisuke Kojiro $^{2} \cdot$ Yuzo Furuta $^{2}$
}

Received: 5 December 2017 / Accepted: 25 April 2018 / Published online: 18 May 2018

(C) The Japan Wood Research Society 2018

\begin{abstract}
This study is focused on what factor mainly affects the mechanical properties of each wood species in the lateral direction. At first, the influence of the density which is closely related to mechanical properties in the longitudinal direction was also researched in the lateral direction. Thus, the elastic modulus, strength, and failure strain in the lateral tension were measured using thin cross-sectional samples of softwoods and hardwoods, having wide varieties in the density and anatomical features. The results obtained are as follows. The linear relationship between the density and the elastic modulus which has been verified in the longitudinal direction was not observed in the lateral tension for the samples with the annual ring inclination of $90^{\circ}$, which samples were influenced by ray arranged parallel to the tensile direction. However, samples with the annual ring inclination of $45^{\circ}$ showed the high correlation between the density and the elastic modulus due to the shearing deformation of the cell shape. On the other hand, the proportional relationship between the elastic modulus and strength which has been verified in the longitudinal direction was not observed in the lateral tension except for the samples with the annual ring inclination of $90^{\circ}$. From the results obtained, it was revealed that the mechanical properties of wood in the lateral direction were significantly affected not only by the density but also by the structural features such as deformation of cell shapes, arrangement of ray or vessels, and the degree of the transition from the earlywood to the latewood.
\end{abstract}

Keywords Lateral tension $\cdot$ Density $\cdot$ Anatomical feature $\cdot$ Cell deformation $\cdot$ Annual ring

\section{Introduction}

Wood shows quite different characteristics in mechanical properties depending on the types of stresses given by bending, compression, tension or shearing, and depending on the load directions such as longitudinal, tangential, and radial, because wood has anisotropic properties.

In the longitudinal direction, there is a tendency that the elastic modulus increases with increasing the density not only within the same species but also between different species of wood. It was reported that the linear relationship was observed between elastic modulus and density [1]. Moreover, it was also reported that the law of mixture, expressed

Yuka Miyoshi

ymiyoshi@ffpri.affrc.go.jp

1 Forestry and Forest Products Research Institute, 1 Matsunosato, Tsukuba, Ibaraki 305-8687, Japan

2 Division of Environmental Sciences, Graduate School of Life and Environmental Sciences, Kyoto Prefectural University, Hangi-cho, Shimogamo, Sakyo-ku, Kyoto 606-8522, Japan by the exponential or the logarithmic function which is calculated based on the complex structure for the solid of cell wall and the void, is applied to the relationship between the elastic modulus and density [2].

On the other hand, the influence of the structural factors on the elastic modulus in the lateral direction is more complicated than that in the longitudinal direction, depending on the shape and arrangement of cells [3-5], the amount of ray [6], and the annual ring angle [7-11]. However, most of the previous studies on the lateral mechanical properties concerning about these structural factors have been progressed in the analysis of the porous structure on the model of wood [12-16] and the researches using a single wood species [3-11]. Therefore, there has been no study for quantitatively showing what factor affects mainly the mechanical properties in the lateral direction of each wood species, then comparing the mechanical characteristics between wood species.

Meanwhile, to clarify the influences of rheological properties and the anatomical feature on the deformation and fracture properties in the lateral tension of wood, tensile and creep tests were performed in water at various temperatures 
using the thin cross-sectional samples of hinoki having different angles of the annual rings [17]. Then, the failure strain, elastic modulus, strength, and creep compliance were calculated from the stress-strain curves and the creep curves obtained. From the results, the arrangement of the ray and the shearing deformation of cells largely affected the lateral tensile deformation properties. The reason why these results were obtained is considered as follows. In the present method, it is possible to obtain the information about the deformation of cells in the lateral direction sensitively because samples with $0.1 \mathrm{~mm}$ thickness in the longitudinal direction were used. Furthermore, in the case of hinoki, the sample with $0.1 \mathrm{~mm}$ thickness in the longitudinal direction has 1 or 2 rays arranged in line. Therefore, if the sample is given the stress until broken, the influence of the weak point such as ray on the mechanical property in the lateral direction is clearly obtained. In addition, it is possible to know the weak point by observing the fracture surface and obtain the results reflecting the reliable strength data because only the samples fractured at their central portion between chucks are adopted to the results. The moisture and temperature conditions of each sample were also precisely made uniform due to the thinness of each sample in the longitudinal direction. From the reasons above, if the lateral tensile test of various wood species is performed in accordance with the method, we can quantitatively clarify the influences of density, shape, and arrangement of cells and structure of the annual rings on the mechanical properties of wood.

This study is focused on what factor mainly affects the mechanical properties of each wood species in the lateral direction. At first, the influence of density which is closely related to mechanical properties in the longitudinal direction was researched also in the lateral direction. Thus, elastic modulus, strength, and failure strain in lateral tension were measured using thin cross-sectional samples of softwoods and hardwoods, having wide varieties in density and anatomical features. For the results of the mechanical properties which show no relation in the density, it was discussed that what structural factors affect the mechanical properties in the lateral direction from the view of the structural features of each wood species.

\section{Materials and methods}

\section{Samples}

The samples of softwood used were sugi (Cryptomeria japonica), hinoki (Chamaecyparis obtusa), icho (Ginkgo biloba), akamatsu (Pinus densiflora), and kaya (Torreya nucifera). The samples of hardwood used were yamaguruma (Trochodendron aralioides), buna (Fagus crenata), hoonoki (Magnolia obovata), keyaki (Zelkova serrata), and arakashi (Quercus glauca). The densities of these wood species used in this study are shown in Fig. 1. Each density was measured at oven-dried condition. The annual ring width and the features of each sample are as follows. For the samples of softwood, the annual ring widths of sugi, hinoki, icho, and akamatsu were $1.8,1.1,7.4$, and $1.6 \mathrm{~mm}$, respectively. The abrupt transition from earlywood to latewood was observed in sugi and akamatsu, but the transition was gradual in hinoki, icho, and kaya. The softwood samples have uniseriate rays. For the samples of hardwood, the annual ring widths of yamaguruma, buna, hoonoki, keyaki, and arakashi were $0.8,1.7,1.7$, 2.0 , and $3.0 \mathrm{~mm}$, respectively. Yamaguruma is vesselless wood, buna and hoonoki are diffuse-porous wood, keyaki is ring-porous wood, and arakashi is radial-porous wood. The hardwood samples have multiseriate ray.

Wood shows different mechanical properties depending on the loading direction [7-11, 17]; therefore, three kind of samples with different annual ring inclinations respect to the tensile direction were used. The block-shaped samples with the annual ring inclinations of $0^{\circ}$ (tangential direction), $45^{\circ}$ (angle between tangential and radial directions), and $90^{\circ}$ (radial direction) against the tensile direction were collected from almost the same growth ring band peripheral to the heart wood. Cross-sectional samples with the thickness of about $0.1 \mathrm{~mm}$ (longitudinal direction) and with the dimensions of $3.4 \mathrm{~mm}$ in width and $20 \mathrm{~mm}$ in length were used for the tensile test (Fig. 2). These thin samples were sliced by sliding microtome with extreme care not to generate scratches or knife marks which could be the origin of fracture. All samples were soaked in water over 3 weeks at $20{ }^{\circ} \mathrm{C}$ and measured in water-saturated condition.

Among the samples, only for icho and akamatsu, we could not make thin samples because the rays were too weak. Therefore, the measurements of icho and akamatsu could not be performed.

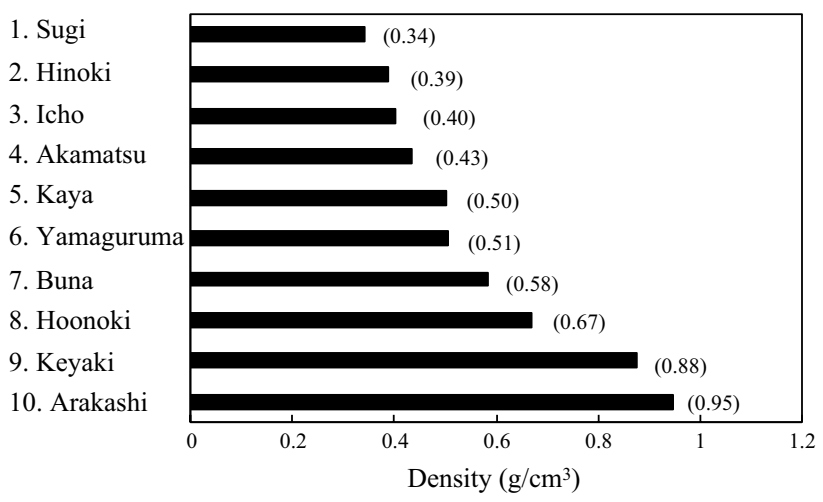

Fig. 1 Density of the various wood species used in measurement 


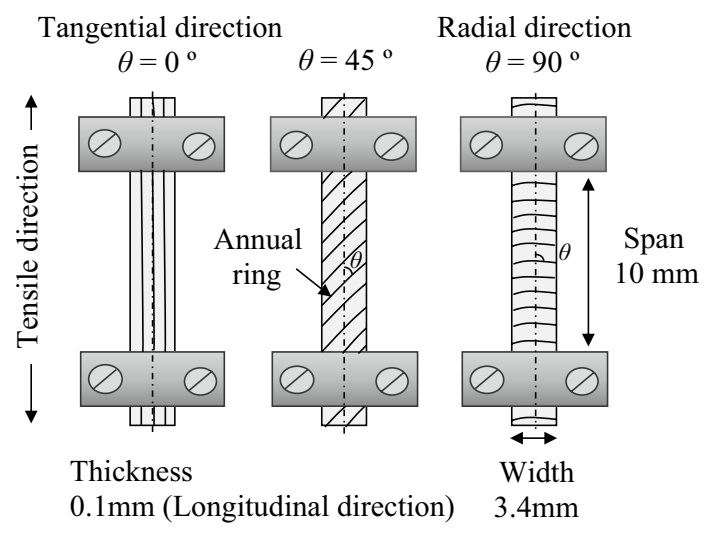

Fig. 2 Schematic diagram of the experimental setup for tensile test

\section{Lateral tensile test}

A thermo-mechanical analyzer (manufactured by Seiko Instruments Co., Ltd.; TMA/SS6100) was used in the mechanical tests. All of the measurements were carried out in water kept at the constant temperature, and the temperatures were 25 and $80^{\circ} \mathrm{C}$. The distance between chucks was set to $10 \mathrm{~mm}$, and the strains of the samples were measured with the increasing load of $1 \mathrm{~N} / \mathrm{min}$ until they were fractured. The measurement results were adopted only for the samples fractured around their central portion, and the samples had exceptional deformation behaviors that were excluded. The number of the samples in the results was adopted 5 per condition. The failure strain was shown as a percentage of the value obtained by dividing elongation before fractured by the chuck distance measured with the minimum load in the device.

\section{Results and discussion}

\section{Effects of density on the mechanical properties in lateral tension}

The effects of density on the elastic modulus, strength, and failure strain are shown in Fig. 3. Regression lines are observed for the results with contribution ratios of 0.30 or more. The sample of buna had a very low strength at $80{ }^{\circ} \mathrm{C}$ and was difficult to measure; therefore, only the result at $25^{\circ} \mathrm{C}$ is shown in the Figures. The elastic modulus at $25{ }^{\circ} \mathrm{C}$ was larger than those at $80{ }^{\circ} \mathrm{C}$ in all samples. The linear relationship between the density and the elastic modulus which has been verified in the longitudinal direction [1] was not observed in the samples with the annual ring inclination of $90^{\circ}$. The elastic modulus of the samples with the annual ring inclination of $90^{\circ}$ was the largest among all the samples, because ray arranged parallel to the tensile direction disturbed the deformation. For the samples with the annual ring inclination of $0^{\circ}$, low correlation was observed in the samples measured at $25^{\circ} \mathrm{C}$. It is confirmed that the elastic modulus of arakashi being radial-porous arrangement measured at $25^{\circ} \mathrm{C}$ was significantly lower than the regression line. On the other hand, the samples with the annual ring inclination of $45^{\circ}$ showed high correlations between the density and the elastic modulus at both temperatures. The cells in the thin cross-sectional samples of wood are deformable, and the shearing deformation is largely caused in the samples with the annual ring inclination of $45^{\circ}$ under the tension [3-5]. The reason why the high correlation was obtained mainly in the samples with $45^{\circ}$ was considered that the low-density wood with thin cell walls was easy to deform the cell shape by shearing stress.

It is considered that the strength is depended on the weakest part in the specimen. Therefore, if there are no defective parts or stress-concentrating parts in the specimens, the strength increases with the increase of the density. However, the correlation between the density and the strength was not observed for the samples with the annual ring inclination of $45^{\circ}$ at both temperatures and inclination of $0^{\circ}$ at $25^{\circ} \mathrm{C}$. For the samples with the annual ring inclination of $0^{\circ}$, although the variation of the strength was large among the wood species at $25^{\circ} \mathrm{C}$, the strength at $80^{\circ} \mathrm{C}$ was roughly aligned. This tendency may suggest that the influence of the structural factors on the strength is remarkable under the low temperature. The low correlation between the density and the strength was observed in the samples with the annual ring inclination of $90^{\circ}$. The ray is arranged parallel to the tensile direction in the samples with the annual ring inclination of $90^{\circ}$. It is also expected that the strength is affected by the ray because the strength of the samples with the annual ring inclination of $90^{\circ}$ is the largest among all the samples. However, from the results of the strength measured at $25^{\circ} \mathrm{C}$, there was a plot of yamaguruma located far above the regression line despite the low density and a plot of keyaki located far below the regression line despite the high density. Furthermore, kaya being the uniseriate ray and keyaki being the multiseriate ray showed the comparable strength; even the difference of the density was large between the species. These results indicate that the strength of the samples with the annual ring inclination of $90^{\circ}$ cannot be explained by only the structural features of the ray. Therefore, the reason why the strength of keyaki measured at $25^{\circ} \mathrm{C}$ was extremely low is considered as follows. In the case of keyaki being ring-porous arrangement, observing the fracture surface in the cross section, the fractures along the vessel arranged in a direction orthogonal to the tensile direction were observed in this study. Similar tendency has been also obtained in the previous study [10]. Therefore, it was considered that the strength tended to be low even though the density was high because the stress 


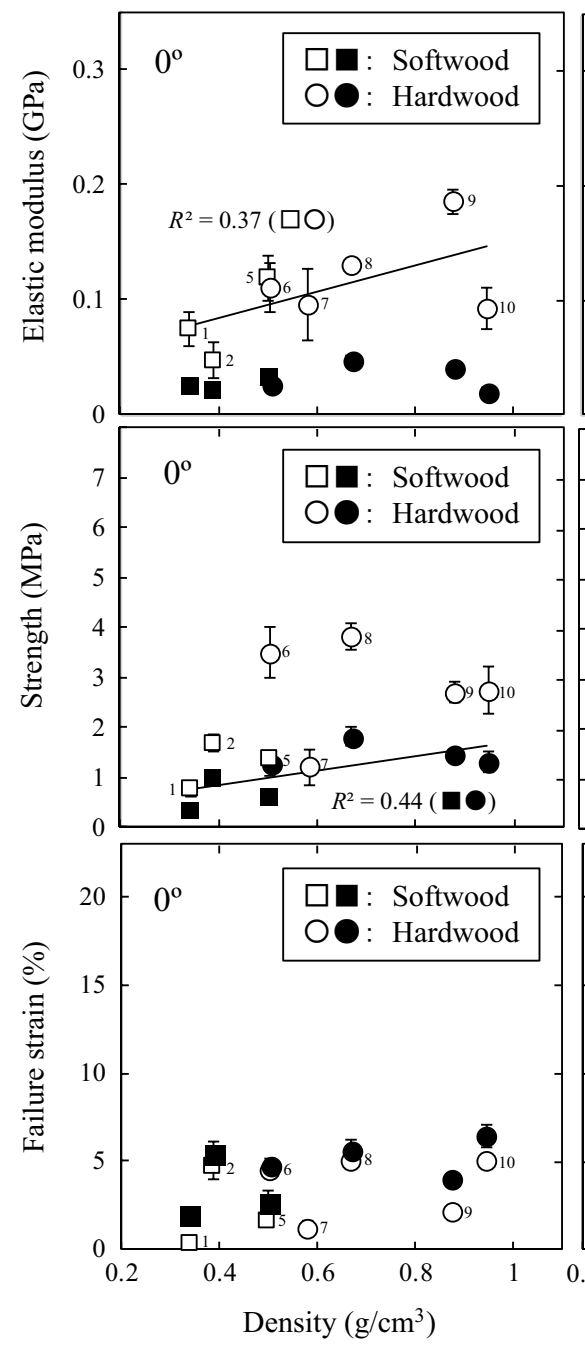

Fig. 3 The effects of density on elastic modulus, strength, and failure strain of the samples with the annual ring inclinations of $0^{\circ}, 45^{\circ}$, and $90^{\circ}$ in the lateral tension. The numbers mean wood species, 1: sugi, 2: hinoki, 5: kaya, 6: yamaguruma, 7: buna, 8: hoonoki, 9: keyaki, and 10: arakashi. The numbers are added beside the plots in the result

was concentrated at the vessels arranged in the direction orthogonal to the tensile direction.

The dependence of the density on the failure strain was not observed in the samples with the annual ring inclinations of $0^{\circ}$ and $90^{\circ}$. However, for the samples with the annual ring inclination of $45^{\circ}$ measured at $80^{\circ} \mathrm{C}$, low correlation between the density and the failure strain was observed, though it was quite small. This indicated that the sample with higher density showed the lower failure strain. The cause of these results is considered as follows. In the case of the samples with low density being the thin cell wall, it is easy to deform the cell shape by shearing stress. If the cells deform easily under the lower stress, it will be possible to increase the amount of deformation of either temperature. Open plots, samples measured at $25^{\circ} \mathrm{C}$; filled plots, samples measured at $80{ }^{\circ} \mathrm{C} . R^{2}$ mean contribution ratio and error bars indicate $95 \%$ confidence interval. The symbols in round brackets beside $R^{2}$ mean the symbols with linear regression

before fracture. Furthermore, the amount of deformation increases due to the reduction of the cell lumen caused by shearing stress. Therefore, in the case of the high-density wood, a large deformation hardly occurs since the volume of the void is small.

On the other hand, the interesting point in this result is that the failure strain of sugi with low density is extremely lower than that of hinoki with similar density in the samples with the annual ring inclination of $45^{\circ}$ at each temperature. From the results of the strength, the strength of sugi was also significantly smaller than that of hinoki. The transition from the earlywood to the latewood is abrupt in sugi [18]. It is considered that this anatomical feature affected the reduction of the strength and the failure strain. 


\section{Relationships between elastic modulus and strength or failure strain}

It is generally known that the relationship between the elastic modulus and the strength is proportional. However, this relation is recognized mainly in the longitudinal direction. Therefore, the relationship between the elastic modulus and the strength was discussed in the lateral tension in Fig. 4. Except for the samples with the annual ring inclination of $90^{\circ}$, clear correlations were not found at 25 and $80^{\circ} \mathrm{C}$. In the samples with the annual ring inclination of $45^{\circ}$, there was no regularity in the distribution of the values. This result suggests that the lateral mechanical properties are significantly affected by wood anatomical structure. On the other hand, the samples with the annual ring inclination of $90^{\circ}$
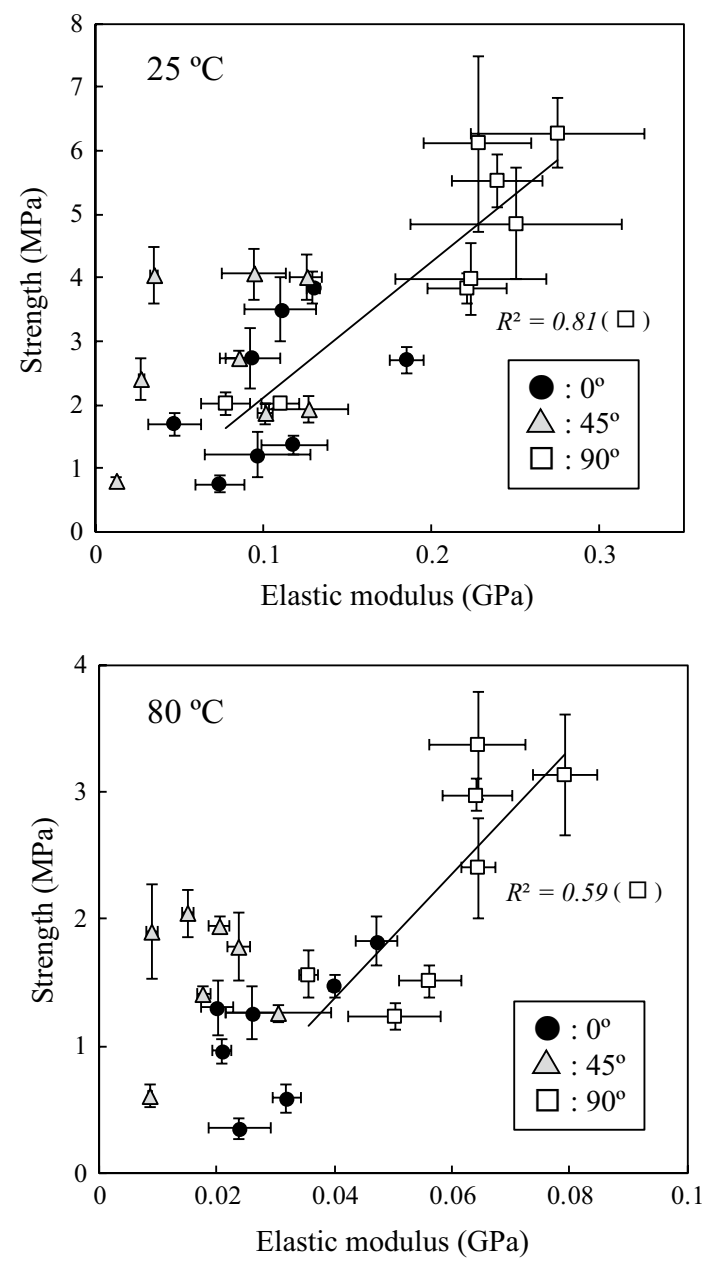

Fig. 4 Relationship between elastic modulus and strength at 25 and $80^{\circ} \mathrm{C}$ in lateral tension. Circles, samples with the annual ring inclination of $0^{\circ}$; triangles, samples with the annual ring inclination of $45^{\circ}$; squares, samples with the annual ring inclination of $90^{\circ} . R^{2}$ mean contribution ratio and error bars indicate $95 \%$ confidence interval. The symbols in round brackets beside $R^{2}$ mean the symbols with linear regression showed the relatively high correlation at each temperature. From these results, in the samples related to the mechanical properties of the ray, the high-density wood with stiff ray has higher elastic modulus and strength.

The relationship between the elastic modulus and the failure strain is shown in Fig. 5. From the figure, the distribution of values was various. For the samples with the annual ring inclination of $90^{\circ}$ which is greatly affected by the ray, the failure strain tended to increase with increasing the elastic modulus, mainly on the results of the hard wood with high elastic modulus. Especially in the samples with the annual ring inclination of $45^{\circ}$, the low correlation was observed at each temperature and the failure strains decreased with increasing the elastic modulus. As a result of the strength mentioned above, there was no relationship between the
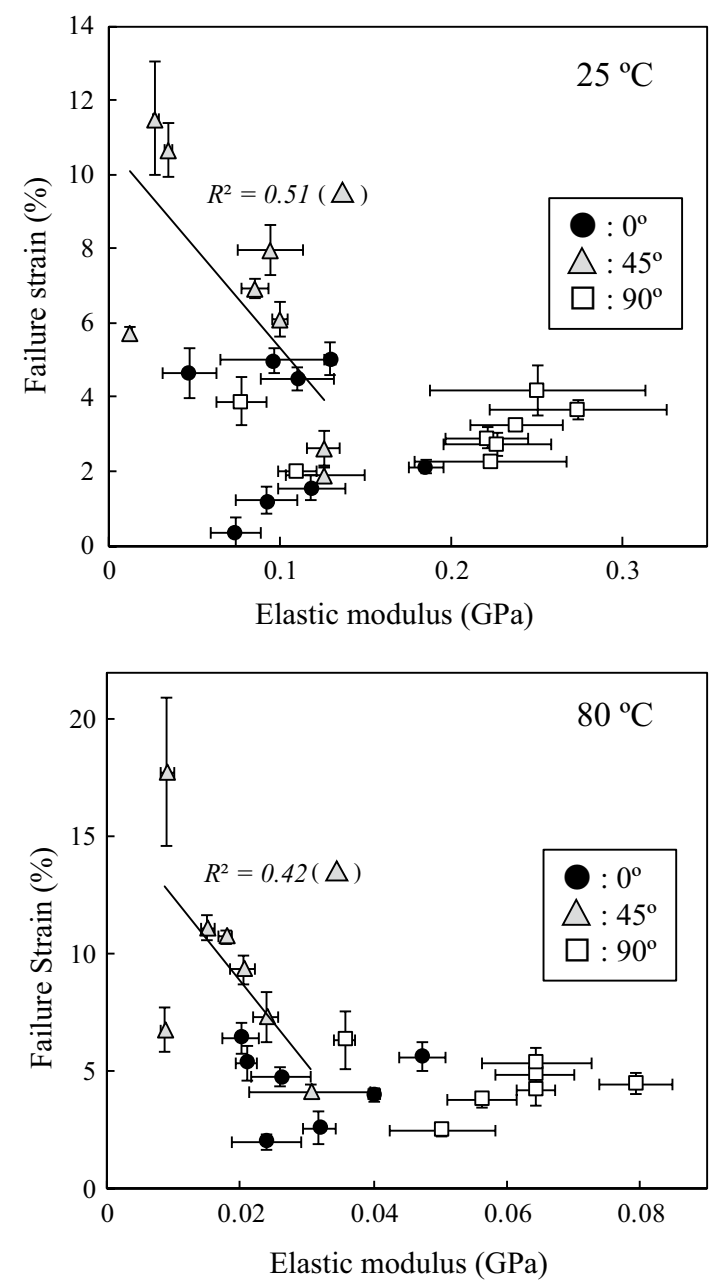

Fig. 5 Relationship between elastic modulus and failure strain at 25 and $80{ }^{\circ} \mathrm{C}$ in lateral tension. Circles, samples with the annual ring inclination of $0^{\circ}$; triangles, samples with the annual ring inclination of $45^{\circ}$; squares, samples with the annual ring inclination of $90^{\circ} . R^{2}$ mean contribution ratio and error bars indicate $95 \%$ confidence interval. The symbols in round brackets beside $R^{2}$ mean the symbols with linear regression 
elastic modulus and the strength in the samples with the annual ring inclination of $45^{\circ}$. Therefore, it is considered that the amount of deformation before fracture may be large in the samples with the annual ring inclination of $45^{\circ}$ with low elastic modulus because the samples can easily deform even with small stress and the strength is not decreased.

However, there was a plot located far below the regression line despite the low density, and this plot was the sample of sugi. These results also suggest that the mechanical property in the lateral tension of sugi may be affected by the factors different from other species such as the distribution of the density of the earlywood and the latewood in an annual ring.

\section{Conclusions}

In this study, to clarify the influence of the density on the lateral mechanical properties of various wood species, elastic modulus, strength, and failure strain in the lateral tension were measured. The linear relationship between the density and the elastic modulus which has been verified in the longitudinal direction was not observed in the lateral tension for the samples with annual ring inclination of $90^{\circ}$, which samples were influenced by ray arranged parallel to the tensile direction. However, the samples with the annual ring inclination of $45^{\circ}$ showed high correlation between the density and the elastic modulus due to the shearing deformation of the cell shape. On the other hand, proportional relationship between the elastic modulus and the strength which has been verified in the longitudinal direction was not observed in the lateral tension except for the samples with the annual ring inclination of $90^{\circ}$. From the results obtained, it was revealed that the mechanical properties of wood in the lateral direction were significantly affected not only by the density but also by the structural features such as deformation of cell shapes, arrangement of ray or vessels, and the degree of the transition from the earlywood to the latewood.

\section{References}

1. Takemura T (1995) Force and wood. In: Fushitani M (ed) Physics of wood (in Japanese). Buneido Publishing, Tokyo, pp 105-106
2. Sobue N (2007) Force and wood. In: Norimoto M (ed) Physics of wood (in Japanese). Buneido Publishing, Tokyo, pp 106-107

3. Fujita S (1971) Studies on the drying check VII. Effect of annual ring orientation on tensile strength perpendicular to grain by microscopical techniques (in Japanese). Mokuzai Gakkaishi 17:28-33

4. Fujita S (1972) Studies on the drying check VIII. Deformation of structural layer during transverse tensile test (in Japanese). Mokuzai Gakkaishi 18:427-433

5. Kanaya N, Yamada T (1967) Wood by transverse tensile tests (in Japanese). Wood Res 41:47-62

6. Schniewind AP (1959) Transverse anisotropy of wood: a function of gross anatomic structure. For Prod J 9:350-359

7. Okuyama T, Suzuki S, Terazawa S (1977) Effect of temperature on orthotropic properties of wood I. On the transverse anisotropy in bending (in Japanese). Mokuzai Gakkaishi 23:609-616

8. Suzuki S, Okuyama T, Terazawa S (1979) Effect of temperature on orthotropic properties of wood II. Proportion of transwall failure. Mokuzai Gakkaishi 25:177-183

9. Miyoshi Y, Kojiro K, Furuta Y (2014) Deformation properties of wood in lateral tension effect of tensile direction to the annual rings, moisture, and temperature on lateral tensile deformation of hinoki (Chamaecyparis obtusa) (in Japanese). Mokuzai Gakkaishi 60:241-248

10. Miyoshi Y, Kojiro K, Furuta Y (2015) Effect of anatomy and thermal-softening properties on lateral tensile deformation properties of various wood species (in Japanese). J Soc Mat Sci Jpn 64:356-361

11. Clauß S, Pescatore C, Niemz P (2014) Anisotropic elastic properties of common ash (Fraxinus excelsior L.). Holzforschung 68:941-949

12. Ohgama T, Yamada T (1971) Porous structure of wood and its relaxation modulus (in Japanese). J Soc Mater Sci 20:1194-1200

13. Ohgama T, Yamada T (1975) Relaxation modulus of wood as a porous material (in Japanese). J Soc Mater Sci 24:873-878

14. Ohgama T, Morooka T, Yamada T (1981) Elastic modulus of wood as a porous material (variation with direction relative to annual ring) (in Japanese). J Soc Mater Sci 30:703-706

15. Ohgama T, Yamada T (1981) Young's moduli of earlywood and latewood in transverse direction of softwood (in Japanese). J Soc Mater Sci 30:707-711

16. Patera A, Carl S, Stampanoni M, Derome D, Carmeliet J (2018) A non-rigid registration method for the analysis of local deformations in the wood cell wall. Adv Struct Chem Imaging 4:1-11

17. Miyoshi Y, Furuta Y (2016) Rheological consideration in fracture of wood in lateral tension. J Wood Sci 62:138-145

18. Japanese Wood Research Society, Wood Anatomy and Wood Property Research Group (2006) IAWA list of microscopic fractures for softwood identification (in Japanese). Kaiseisha Press, Shiga. pp 6-8 